\title{
The Effects of Incubation Length and Temperature on the Specificity and Sensitivity of the BANA (N-Benzoyl- DL-Arginine-Naphthylamide) Test
}

\author{
Joseph Amalfitano, * Anna B. De Filippo, ${ }^{\dagger}$ Walter A. Bretz, ${ }^{\ddagger}$ and Walter J. Loesche, ${ }^{*}$
}

A PREVIOUS MULTI-CENTER STUDY EXAMINING patients diagnosed as having at least four periodontally diseased teeth showed that when BANA (N-Benzoyl-DL-Arginine-Naphthylamide) hydrolysis by periodontal pathogens such as Treponema denticola, Porphyromonas gingivalis, and Bacteroides forsythus was evaluated versus clinical parameters such as clinical judgment of disease, bleeding on probing, and pocket depth, the sensitivity of the test was $84 \%, 82 \%$, and $87 \%$, respectively, while the specificity was only $42 \%, 41 \%$, and $32 \%$, respectively. The purpose of the present investigation was to improve the specificity of the test while retaining a high level of sensitivity in both gingivally healthy and periodontally diseased groups. One hundred forty-nine patients participated in this study providing 3,497 interproximal plaque samples. Gingival health was measured using the papillary bleeding score and this was compared with the presence or absence of detectable trypsin-like activity, as determined by the BANA hydrolysis of interproximal plaque samples, using a commercially-available test. Sensitivity and specificity were measured by varying the incubation time and temperature of the enzymatic assay. Using the correlated binomial model to analyze site-specific data within a patient, the specificity was highest at $35^{\circ} \mathrm{C}$ and 5 minutes incubation $(94 \%)$, and lowest at $45^{\circ} \mathrm{C}$ and 15 minutes incubation (33\%). Sensitivity was highest at $45^{\circ} \mathrm{C}$ or $55^{\circ} \mathrm{C}$ and 15 minutes incubation $(90 \%)$ and lowest at $35^{\circ} \mathrm{C}$ and 5 minutes incubation $(47 \%)$. When the sensitivity and specificity of the test run at $35^{\circ} \mathrm{C}$ for 5 minutes were used in a model for a diagnostic test proposed by Douglass and Fox, the test has utility when used on older general dental patients and for periodontal patients. $J$ Periodontol 1993; 64:848-852.

Key Words: Periodontal diseases/diagnosis; periodontal diseases/pathogenesis; dental plaque; dental plaque index.

Porphyromonas gingivalis, Treponema denticola, and Bacteroides forsythus are frequently associated with periodontal disease $^{1,2}$ and possess in vivo an enzyme capable of hydrolyzing the synthetic trypsin substrate, N-benzoyl-DL-arginine-2-naphthylamide (BANA). This enzyme is detectable when only $10^{4}$ colony forming units (CFU) of these species are present in the plaque sample. ${ }^{3}$ We have now screened over 60 species of plaque bacteria (a total of over 300 strains) for the presence of the BANA enzyme and only these three species were uniformly BANA-positive. ${ }^{4} \mathrm{Six}$

*Department of Biologic and Materials Sciences, University of Michigan, School of Dentistry, Ann Arbor, MI.

${ }^{\dagger}$ Program in Periodontics, University of Toronto, Faculty of Dentistry, Toronto, ON.

‡Department of Cariology and General Dentistry, University of Michigan, School of Dentistry.

${ }^{\S}$ Department of Microbiology and Immunology.
Bacteroides and Capnocytophaga species on occasion (36 of 152 separate determinations) gave a weakly positive or positive BANA reaction, even when very large numbers of CFUs were used; i.e., $>10^{7} \mathrm{CFU} .{ }^{4}$ Subsequently, we have found that Pseudomonas aeruginosa, Pseudomonas fluorescens, and Rothia dentocariosa can hydrolyze the BANA substrate, but these species are rarely isolated from subgingival plaque samples.

These considerations indicate that the detection of the BANA enzyme in plaque samples could serve as a marker for the presence of $P$. gingivalis, $T$. denticola, and $B$. forsythus in these plaque samples. This possibility was supported by the finding that in over 200 plaque samples removed from diseased sites, the BANA test results were indistinguishable from results obtained with specific DNA probes and specific immunological reagents for $T$. denticola, $P$. gingivalis, and $B$. forsythus. ${ }^{3}$ 
A previous multi-center study ${ }^{5}$ examining patients who had at least four teeth diagnosed as periodontally diseased has shown that when BANA hydrolysis was measured using a commercially-available solid state assay" and compared to immunological reagents for $T$. denticola and $P$. gingivalis (ELISA) as the reference test, the sensitivity was $92 \%$, the specificity was $70 \%$, and the accuracy was $84 \%$. In the same study, when the test was evaluated versus parameters such as clinical judgment of disease, bleeding on probing, and pocket depth, the sensitivity was $84 \%, 82 \%$, and $87 \%$ respectively, while the specificity was only $42 \%, 41 \%$, and $32 \%$ respectively. Similar sensitivities and specificities were found when the immunologic reagents were compared to the same clinical parameters. The agreement between the immunological data and assay results indicated that both procedures were detecting the BANA-positive species when they were present in the plaque samples. However, the low specificities indicated that the BANA organisms can be present in plaque samples when there is no clinical disease.

The aim of the present study was to improve the overall accuracy of the test by improving the specificity. Because an enzyme test is influenced by the temperature and length of incubation, we varied the length and temperature of incubation and determined how these changes would affect the specificity, sensitivity, and accuracy when compared to clinical parameters. In this investigation, we compared the assay results either $35^{\circ}, 45^{\circ}$, or $55^{\circ} \mathrm{C}$ after 5 or 15 minutes of incubation with the clinical status of plaque samples removed from either periodontally-diseased patients or from periodontally-healthy subjects.

\section{MATERIALS AND METHODS}

\section{Study Groups}

The periodontally-healthy group was described earlier. ${ }^{6}$ Briefly, 90 medically/healthy patients with no periodontal pockets greater than or equal to $4 \mathrm{~mm}$ were recruited from students and volunteers at the University of Michigan, School of Dentistry. These subjects were not completely free of gingivitis (See Fig. 1A), but for convenience are referred to as periodontally healthy. The diseased group consisted of 59 patients with at least 4 periodontal probing sites $\geq 6$ $\mathrm{mm}$ who were recruited from subjects who presented at a Brazilian Dental Clinic in Rio de Janeiro, Brazil. These populations were convenience samples and were chosen to have two extreme populations in regard to periodontal health. Previous studies of the assay in the U.S. ${ }^{5}$ and in Brazil ${ }^{7}$ have shown comparable results. None of the subjects reported using antibiotics in the 3-month period prior to the clinical examination.

\section{Clinical Examination}

Pocket depths were measured at 6 sites per tooth using the Michigan $\mathrm{O}$ probe. Gingival health was determined using

"PerioScan, Oral B Laboratories, Redwood City, CA. the papillary bleeding score, $\mathrm{PBS},{ }^{8}$ in which a separate wooden toothpick" was inserted into all interproximal sites, excluding the area between the second and third molars. A patient could have a maximum of 26 sampled sites. The degree of bleeding (PBS) was scored as follows:

$0=$ no bleeding, gingival color and contour appear to be healthy; $1=$ no bleeding gingiva is slightly inflamed and/or slightly red; 2 = slight bleeding or spotting of blood, no flow along the gingival margin; 3 = bleeding, blood flow remains confined to the area of the papilla; $4=\mathrm{im}$ mediate bleeding, blood flow is copious and spreads to the adjacent teeth; $5=$ tissue is grossly inflamed, hemorrhagic and edematous.

Two calibrated examiners (WB and WL) collected the U.S. data and two calibrated examiners (WB and ADeF) collected the Brazilian data. One examiner (WB) was common to both collection sites.

After the PBS was determined for each site, a toothpick was wiped on the BANA-impregnated filter strip that ran along the lower border of the card. An upper reagent strip containing Evan's black dye was activated by dampening the strip with water. The lower strip was then folded over the upper strip so that any naphthylamide released from the BANA-impregnated strip would diffuse into the upper strip where it could react with the Evan's black dye, forming a permanent blue-black color. After folding, the cards were inserted into heating blocks and incubated at the appropriate temperature. Patients were randomly assigned into one of three groups and cards were incubated at either $35^{\circ}, 45^{\circ}$, or $55^{\circ} \mathrm{C}$. Cards from all groups were removed at 5 minutes, scored for BANA activity and then reincubated in the same heating block for an additional 10 minutes. A score of 0 was assigned when no blue color was visible; a score of 1 was assigned when a faint blue color was observed, and a 2 was scored when a definite blue color was observed. The actual area of color was not as important as the intensity of color. For statistical analysis, BANA scores of 1 and 2 were combined as positive results.

\section{Statistical Analysis}

Data including age, gender, PBS, and assay results at 5 and 15 minutes at each interproximal site were recorded on standardized entry forms, and entered into a database file. Statistical parameters including sensitivity, specificity, and accuracy were calculated by a correlated binomial model, ${ }^{9}$ using the assay result as the test procedure and the clinical condition as the reference procedure. A site was considered diseased if it had a PBS $\geq 2$. A healthy site had a PBS $\leq$ 1. A true positive was recorded when a PBS for a particular site was $\geq 2$ and the BANA score was $\geq 1$. A true negative was scored when the PBS was $<2$ and the BANA score was 0 . A false positive was scored when PBS was $<2$ and the BANA score was $\geq 1$. A false negative was scored when PBS was $>2$ and the BANA score was 0 . 
Table 1. Characteristics of Study Groups

\begin{tabular}{|c|c|c|c|c|c|}
\hline Group & Number & Mean Age & Age Range & Females & Males \\
\hline \multicolumn{6}{|l|}{ Healthy* } \\
\hline $35^{\circ} \mathrm{C}$ & 33 & 30.5 & $21-51$ & 11 & 22 \\
\hline $45^{\circ} \mathrm{C}$ & 28 & 30.1 & $22-47$ & 13 & 15 \\
\hline $55^{\circ} \mathrm{C}$ & 29 & 30.6 & $16-55$ & 10 & 19 \\
\hline Total/Average & 90 & 30.4 Average & & 34 & 56 \\
\hline \multicolumn{6}{|l|}{ Diseased $^{\dagger}$} \\
\hline $35^{\circ} \mathrm{C}$ & 20 & 44.1 & $24-70$ & 14 & 6 \\
\hline $45^{\circ} \mathrm{C}$ & 20 & 48.8 & $31-64$ & 12 & 8 \\
\hline $55^{\circ} \mathrm{C}$ & 19 & 47.8 & $29-66$ & 10 & 9 \\
\hline Total/Average & 59 & 46.9 Average & & 36 & 23 \\
\hline
\end{tabular}

*Subjects entered at University of Michigan Dental Clinic.

'Patients entered at the Rio de Janeiro Clinic.

\section{RESULTS}

The demographics of the study groups are summarized in Table 1 . The periodontally-healthy groups had 90 patients with a mean age of 30.4 years with more males than females. The periodontally-diseased groups had 59 patients, were older with a mean age of 46.9 years and had more females than males. The PBS among the two groups are summarized in Figures 1A and 1B. The periodontally-healthy groups had $78 \%$ of the 2,235 interproximal sites scored as healthy; i.e., PBS $\leq 1$. The diseased group had no sites with a PBS of 0 and $88 \%$ of the 1,256 sites were diseased; i.e., PBS $\geq 2$. These two groups, as expected, were extremely different in their periodontal health profiles. They thus provided the opportunity to evaluate the assay, using different incubation formats with plaque samples, than might be representative of those found in the general population.

The frequency of true-positives (sensitivity), true-negatives (specificity), false-positives, and false-negatives among the groups are presented in Table 2. True-negatives were higher in both populations at all temperatures at 5 minutes incubation time compared to 15 minutes incubation time. The opposite was the case for true-positives, which were higher in both groups at all temperatures when the incubation time was extended from 5 to 15 minutes. The numbers of false-positives decreased substantially in both the diseased and healthy groups by reading the tests at $5 \mathrm{~min}$ utes as opposed to 15 minutes, with the exception of the diseased group at $55^{\circ} \mathrm{C}$, which remained unchanged. The numbers of false-negatives dropped in all groups when the test was read at 15 minutes as opposed to 5 minutes.

The specificities, sensitivities, and accuracies, calculated with the correlated binomial model which takes into account site-specific data obtained in a subject, ${ }^{9}$ are presented in Table 3 individually for both the diseased and healthy groups. The healthy population had the highest specificity $(96 \%)$ when the cards were incubated at $35^{\circ} \mathrm{C}$ and read at 5 minutes, whereas the lowest specificity $(45 \%)$ was obtained when the cards were incubated for $55^{\circ} \mathrm{C}$ for 15 minutes. Sensitivity in the healthy group was highest $(84 \%)$ when the cards was incubated at either $45^{\circ}$ or $55^{\circ} \mathrm{C}$ and read at 15 minutes. Sensitivity was lowest in this group; i.e., $22 \%$ at $35^{\circ} \mathrm{C}$ and 5 minutes. The accuracy of the healthy popu-

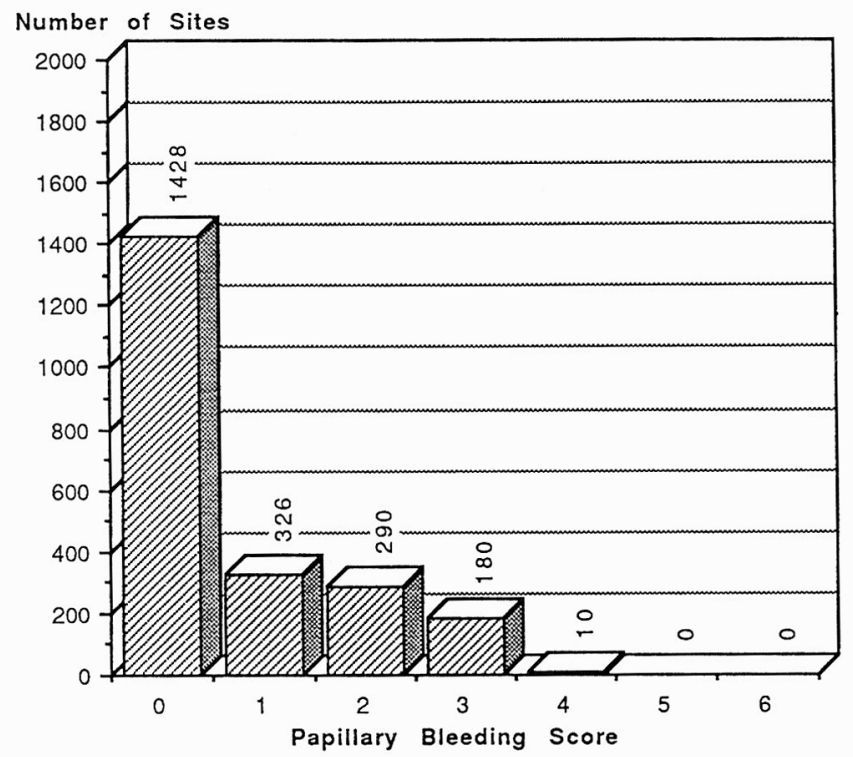

Figure 1A. Distribution of papillary bleeding scores in interproximal sites of periodontally-healthy subjects.

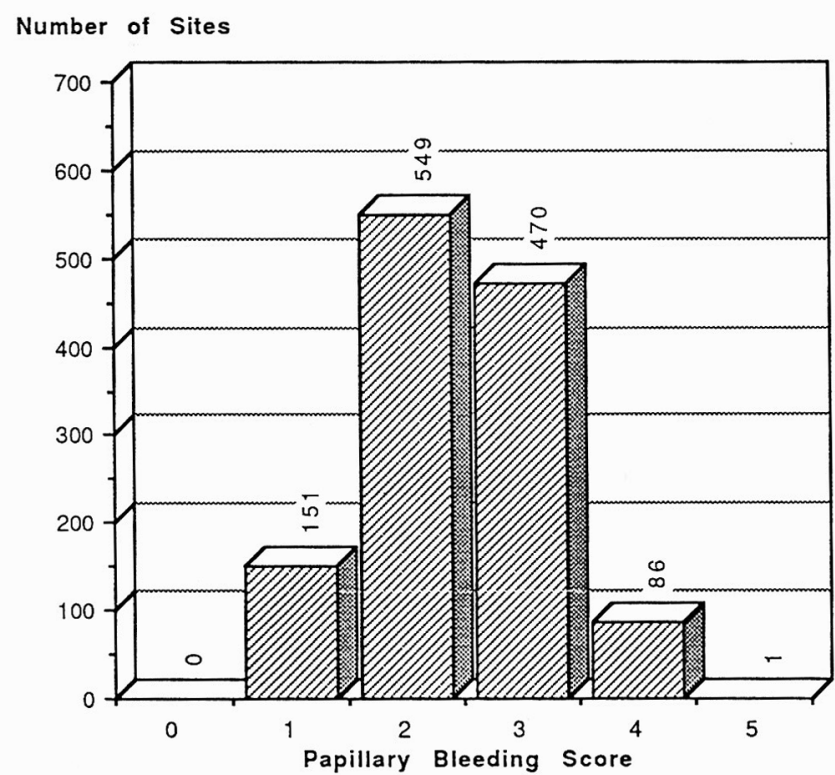

Figure 1B. Distribution of papillary bleeding scores in interproximal sites of periodontally-diseased subjects.

lation was highest $(73 \%)$ when the cards were incubated for $35^{\circ} \mathrm{C}$ for either 5 or 15 minutes.

Specificity of the diseased group was highest $(65 \%)$ when the test was performed at $35^{\circ} \mathrm{C}$ and read at 5 minutes. Specificity was lowest in this group $(6 \%)$ when the cards were incubated at $45^{\circ} \mathrm{C}$ and 15 minutes. The diseased population had the highest sensitivity (93\%) when the cards were incubated at $45^{\circ} \mathrm{C}$ for read at 15 minutes. Sensitivity was lowest in this group when the cards were incubated for $35^{\circ} \mathrm{C}$ for 5 minutes $(60 \%)$. The accuracy of the test in the diseased population was $83 \%$ when the cards were incubated at $45^{\circ} \mathrm{C}$ for 15 minutes and $85 \%$ when incubated at $55^{\circ} \mathrm{C}$ for 15 minutes. 
Table 2. Relationship Between Papillary Bleeding Scores and BANA Results as a Function of Incubation Temperature and Length of Incubation

\begin{tabular}{crrrrr}
\hline Group & $\begin{array}{c}\text { Time } \\
\text { Minutes }\end{array}$ & TN $^{*}$ & FP $^{+}$ & TP $^{*}$ & FN $^{\S}$ \\
\hline Healthy & & & & & \\
$35^{\circ} \mathrm{C}$ & 5 & $586^{\prime \prime}$ & 24 & 46 & 169 \\
& 15 & 445 & 165 & 114 & 101 \\
$45^{\circ} \mathrm{C}$ & 5 & 386 & 192 & 65 & 52 \\
& 15 & 207 & 371 & 98 & 19 \\
$55^{\circ} \mathrm{C}$ & 5 & 403 & 163 & 92 & 57 \\
& 15 & 282 & 284 & 127 & 22 \\
Diseased & & & & & \\
$35^{\circ} \mathrm{C}$ & 5 & 32 & 16 & 207 & 147 \\
& 15 & 16 & 32 & 277 & 77 \\
$45^{\circ} \mathrm{C}$ & 5 & 27 & 25 & 266 & 96 \\
& 15 & 3 & 49 & 337 & 25 \\
$55^{\circ} \mathrm{C}$ & 5 & 8 & 43 & 348 & 42 \\
& 15 & 7 & 44 & 371 & 19 \\
& & & & & \\
\hline
\end{tabular}

*True-negative, $\mathrm{PBS}<2$ and BANA $=0$.

${ }^{\dagger}$ False-positive, $\mathrm{PBS}<2$ and $\mathrm{BANA} \geq 1$.

FTrue-positive, $\mathrm{PBS} \geq 2$ and $\mathrm{BANA} \geq 1$.

${ }^{s}$ False-negative, $\mathrm{PBS} \geq 2$ and $\mathrm{BANA} \leq 1$.

Number of sites.

Table 3. Specificity, Sensitivity, and Accuracy of the Test at Different Temperatures and Length of Incubation

\begin{tabular}{|c|c|c|c|c|}
\hline Group & $\begin{array}{c}\text { Time } \\
\text { Minutes }\end{array}$ & Specificity $(\%)^{*}$ & $\begin{array}{c}\text { Sensitivity } \\
(\%)^{*}\end{array}$ & $\begin{array}{c}\text { Accuracy } \\
(\%)^{*}\end{array}$ \\
\hline \multirow[t]{2}{*}{$\begin{array}{l}\text { Healthy } \\
35^{\circ} \mathrm{C}\end{array}$} & 5 & $96^{\dagger}(94.5-97.5)^{\ddagger}$ & $22(16-28)^{\ddagger}$ & $73(70-76)^{\ddagger}$ \\
\hline & 15 & $75(72-78)$ & $54(47-61)$ & $73(70-76)$ \\
\hline \multirow[t]{2}{*}{$45^{\circ} \mathrm{C}$} & 5 & $64(60-68)$ & $54(45-63)$ & $64(60-68)$ \\
\hline & 15 & $34(30-38)$ & $84(77-91)$ & $46(42-50)$ \\
\hline \multirow[t]{2}{*}{$55^{\circ} \mathrm{C}$} & 5 & $70(66-74)$ & $64(56-72)$ & $69(66-72)$ \\
\hline & 15 & $45(41-49)$ & $84(78-90)$ & $56(52-60)$ \\
\hline \multirow[t]{2}{*}{$\begin{array}{c}\text { Diseased } \\
35^{\circ} \mathrm{C}\end{array}$} & 5 & $65](51-79)$ & $60(55-65)$ & $63(58-68)$ \\
\hline & 15 & $36(22-50)$ & $82(69-95)$ & $71(66-76)$ \\
\hline \multirow[t]{2}{*}{$45^{\circ} \mathrm{C}$} & 5 & $51(37-65)$ & $75(70-80)$ & $73(68-78)$ \\
\hline & 15 & $6(0-12)$ & $93(90-96)$ & $83(79-87)$ \\
\hline \multirow[t]{2}{*}{$55^{\circ} \mathrm{C}$} & 5 & $16(6-26)$ & $89(86-92)$ & $81(77-85)$ \\
\hline & 15 & $14(4-24)$ & $79(75-83)$ & $85(82-88)$ \\
\hline
\end{tabular}

*Specificities, sensitivities, and accuracies calculated with the correlated binomial model. ${ }^{9}$

${ }^{+}$High and low values for group are in boxes.

$\$ 95 \%$ confidence intervals in parentheses.

Table 4 shows the results of a combined analysis of the healthy and diseased populations. Using the correlated binomial model, the specificity was highest at $35^{\circ} \mathrm{C}$ and 5 minutes incubation (94\%) and lowest at $45^{\circ} \mathrm{C}$ and $15 \mathrm{~min}$ utes incubation (33\%). Sensitivity was highest at $45^{\circ}$ or $55^{\circ} \mathrm{C}$ and 15 minutes incubation $(90 \%)$ and lowest at $35^{\circ} \mathrm{C}$
Table 4. Sensitivity, Specificity, and Accuracy of the BANA Test When Diseased and Healthy Groups Are Combined at Various Times and Temperatures

\begin{tabular}{|c|c|c|c|c|}
\hline $\begin{array}{l}\text { Degrees } \\
\text { Celsius }\end{array}$ & $\begin{array}{c}\text { Time } \\
\text { Minutes }\end{array}$ & & $\%$ & $\begin{array}{l}95 \% \text { Confidence } \\
\text { Interval }\end{array}$ \\
\hline \multirow[t]{3}{*}{$35^{\circ}$} & 5 & $\begin{array}{l}\text { Specificity* } \\
\text { Sensitivity* }\end{array}$ & $\begin{array}{l}94 \%^{+} \\
47 \\
\end{array}$ & $\begin{array}{l}92-96^{*} \\
43-51\end{array}$ \\
\hline & & Accuracy* & 70 & $67-73$ \\
\hline & 15 & $\begin{array}{l}\text { Specificity } \\
\text { Sensitivity } \\
\text { Accuracy }\end{array}$ & $\begin{array}{l}73 \\
69 \\
73\end{array}$ & $\begin{array}{l}70-76 \\
66-72 \\
70-76\end{array}$ \\
\hline \multirow[t]{2}{*}{$45^{\circ}$} & 5 & $\begin{array}{l}\text { Specificity } \\
\text { Sensitivity } \\
\text { Accuracy }\end{array}$ & $\begin{array}{l}65 \\
71 \\
67\end{array}$ & $\begin{array}{l}61-69 \\
67-75 \\
64-70\end{array}$ \\
\hline & 15 & $\begin{array}{l}\text { Specificity } \\
\text { Sensitivity } \\
\text { Accuracy }\end{array}$ & $\begin{array}{l}33 \\
90 \\
.52\end{array}$ & $\begin{array}{l}29-37 \\
87-93 \\
48-56\end{array}$ \\
\hline \multirow[t]{4}{*}{$55^{\circ}$} & 5 & $\begin{array}{l}\text { Specificity } \\
\text { Sensitivity }\end{array}$ & $\begin{array}{l}63 \\
80 \\
\end{array}$ & $\begin{array}{l}58-66 \\
76-84\end{array}$ \\
\hline & & Accuracy & 74 & $72-78$ \\
\hline & 15 & $\begin{array}{l}\text { Specificity } \\
\text { Sensitivity }\end{array}$ & $\begin{array}{l}43 \\
90 \\
\end{array}$ & $\begin{array}{l}39-47 \\
87-93\end{array}$ \\
\hline & & Accuracy & 71 & $68-74$ \\
\hline
\end{tabular}

*Correlated binomial model.

tValues in box would be useful as a diagnostic tool in select populations.

and 5 minutes incubation (47\%). Accuracy was highest at $55^{\circ} \mathrm{C}$ and 5 minutes $(74 \%)$ and lowest at $45^{\circ} \mathrm{C}$ and 15 minutes $(52 \%)$.

\section{DISCUSSION}

Previous studies that examined BANA reactions of subgingival plaques among diseased subjects showed that while the sensitivity of the test was high, the specificity was usually low. ${ }^{4}$ This is because the test can detect as few as $10^{4}$ $\mathrm{CFU}$ using the incubation protocol of 15 minutes at $55^{\circ} \mathrm{C}^{3}$ These low levels of organisms reflect colonization within the plaque, but rarely are associated with overt clinical signs of periodontal disease. This protocol had been developed in the laboratory to detect about $10^{6} \mathrm{CFU}$ of in vitro grown cells of $T$. denticola and/or $P$. gingivalis. ${ }^{4}$ However, when plaque samples are used, this protocol will detect about $10^{4}$ CFU of $T$. denticola, $P$. gingivalis, and $B$. forsythus, ${ }^{3}$ a finding which suggests that in vivo, these organisms are forming more copies of the enzyme per CFU. This ability to detect low levels of CFU in vivo means that the BANA test would show a high rate of false-positives relative to the clinical parameters, as the test is detecting colonization (carrier status) as well as infection by these organisms. This can be remedied by making the test less sensitive (in the microbiological sense) so that it will detect fewer organisms at levels $>10^{4} \mathrm{CFU}$. Another potential explanation for the low specificity may be that the test was detecting other species capable of hydrolyzing BANA such as the BANA variable Bacteroides and Capnocytophaga species ${ }^{4}$ and $P$. aeruginosa, $P$. fluorescens, or $R$. dentocariosa. However, 
these latter species are infrequently isolated from subgingival plaque and would not be expected to confound the results.

We have shown in this study that the test results can be optimized for sensitivity and specificity relative to clinical status by varying the incubation time and temperature. Optimal specificity $(94 \%)$ was obtained when the cards were incubated at $35^{\circ} \mathrm{C}$ for 5 minutes, while optimal sensitivity (90\%) was obtained by incubating the cards for 15 minutes at either $45^{\circ} \mathrm{C}$ or $55^{\circ} \mathrm{C}$ (Table 4). The accuracy of the test was highest $(74 \%)$ when the cards were incubated at $55^{\circ}$ for 5 minutes. However, if the optimal performance of the test required running several cards simultaneously at different times and temperatures, this would only confuse matters.

Recently, Douglass and Fox presented a model to determine the value of bacteriologically-based diagnostic tests in periodontal disease. ${ }^{10}$ Crucial to their argument was the decision that "the diagnostic test will have a different predictive value positive and predictive value negative when applied to populations with more or less underlying disease prevalence." Accordingly, they applied their analysis to five populations each with a different prevalence of periodontal disease; i.e., the general population; the population who regularly visits the dentist, i.e., dental utilizers; the population initially diagnosed with periodontal disease; the periodontal maintenance patient; and the refractory periodontal patient. Their theoretical analysis found no diagnostic test that would be helpful in the screening of the general population for putative periodontal pathogens, but did find that a test could be useful among three distinct populations: dental utilizers over the age of 45 years, for newly diagnosed periodontal patients, and for periodontal maintenance patients. They presented a 2-way threshold analysis of sensitivity and specificity listing the values that would be required in each of these three populations for a diagnostic test to be of value.

We have applied their threshold values to those incubation protocols which we found to be optimal for specificity, sensitivity, and accuracy (Table 4). The $35^{\circ} \mathrm{C}, 5$-minute protocol which had a sensitivity of only $47 \%$ would, because of its high specificity, be of diagnostic value in the initially diagnosed periodontal patient, in periodontal maintenance patients, and also in patients who are both $\geq 45$ years and who regularly see their dentist. The $55^{\circ} \mathrm{C} 5$-minute and $55^{\circ} \mathrm{C} 15$-minute protocol would be of value in the initially diagnosed periodontal patient. Thus, in theory, these three incubation protocols of the test could be of diagnostic value in periodontal disease. In practice we have found that one of them, the initially recommended $55^{\circ} \mathrm{C}, 15$-minute protocol, can be used to diagnose an anaerobic infection in the untreated periodontal patient, a usage predicted by the Douglass-Fox model. We had also found that tooth sites that had BANA-positive plaques as obtained with the $55^{\circ} \mathrm{C}$ 15-minute protocol were more likely to lose attachment following treatment than were tooth sites that had BANAnegative plaques. ${ }^{11}$ We have also used this BANA protocol as a means of measuring patient compliance with the unsupervised usage of metronidazole. ${ }^{12}$ The present results suggest that in those circumstances in which some knowledge of the bacterial flora colonizing a plaque would be helpful, the lower temperature and incubation time protocol; i.e., $35^{\circ} \mathrm{C} 5$-minute, would extend the utility of the test to both older dental utilizers as well as to periodontal patients.

\section{Acknowledgments}

This work was supported by a gift from Oral-B Laboratories, Redwood City, CA. Carol Gerlach prepared the manuscript.

\section{REFERENCES}

1. Moore WEC. Microbiology of periodontal disease. $J$ Periodont Res 1987;22:335-341.

2. Loesche WJ, Hujoel PP. Microbiologically based diagnostic tests for periodontitis. Considerations of sensitivity, specificity and accuracy. In: Markers of Disease Susceptibility and Activity for Periodontal Diseases. Johnson N, ed. Cambridge: Cambridge University Press; 1991:417-440.

3. Loesche WJ, Lopatin D, Giordano J, Alcoforado G, Hujoel PP. Comparison of the benzoyl-DL-arginine-naphthylamide (BANA) test, DNA probes, and immunological reagents for ability to detect anaerobic periodontal infections due to Porphyromonas gingivalis, Treponema denticola, and Bacteroides forsythus. J Clin Microbiol 1992;30:42733.

4. Loesche WJ, Bretz WA, Kerschensteiner D, et al. Development of a diagnostic test for anaerobic periodontal infections based on plaque hydrolysis of benzoyl-DL-arginine-naphthylamide. J Clin Microbiol 1990;28:1551-1559.

5. Loesche WJ, Bretz WA, Lopatin DE, et al. Multi-center clinical evaluation of a chairside method for detecting certain periodontopathic bacteria in periodontal disease. J Periodontol 1990;61:189-196.

6. Feitosa ACR, Amalfitano J, Loesche WJ. Effect of incubation temperature on the specificity of the BANA (N-Benzoyl-DL-ArginineNaphthylamide) test. Oral Microbiol Immunol 1993;8:57-61.

7. Bretz WA, Eklund SA, Radicchi R, et al. The use of rapid enzymatic assay in the field for the detection of infections associated with adult periodontitis. J Public Health Dent 1993;(in press).

8. Loesche WJ. Clinical and microbiological aspects of chemotherapeutic agents used according to the specific plaque hypothesis. $J$ Dent Res 1979;58:2404-2412.

9. Hujoel PP, Mouton L, Loesche WJ. Estimation of sensitivity and specificity of site-diagnostic tests. J Periodont Res 1990;25:193-196.

10. Douglass CW, Fox CH. Determining the value of a periodontal diagnostic test. J Periodontol 1991;62:721-730.

11. Loesche WJ, Giordano J, Hujoel PP. The utility of the BANA test for monitoring anaerobic infections due to spirochetes (Treponema denticola) in periodontal disease. $J$ Dent Res 1990;69:1696-1702.

12. Loesche WJ, Grossman N, Giordano J. Metronidazole in periodontitis (IV). The effect of patient compliance on treatment parameters. J Clin Periodontol 1993;20:96-104.

Send reprint requests to: Dr. Walter J. Loesche, University of Michigan, School of Dentistry, Ann Arbor, MI, 48109-1078.

Accepted for publication February 19, 1993. 